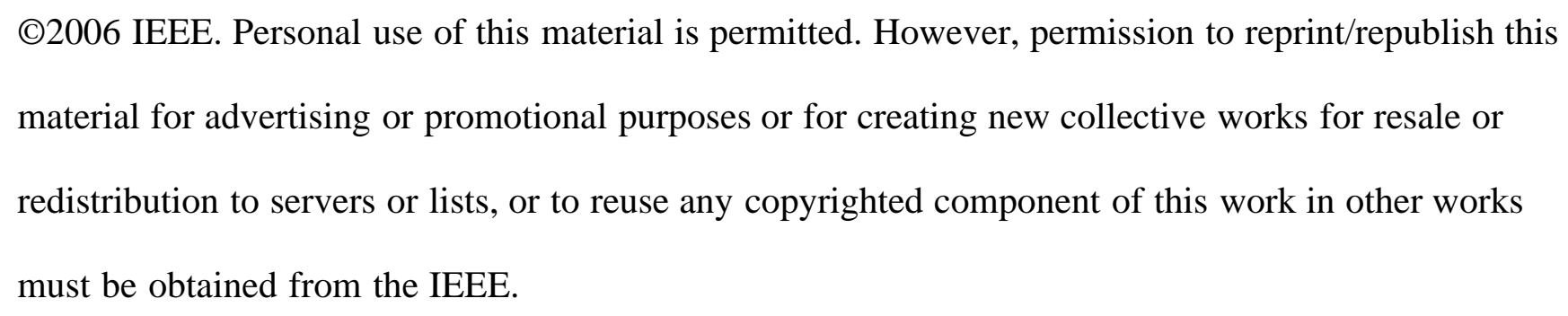




\title{
ROBUST MINIMUM VARIANCE ADAPTIVE BEAMFORMERS AND MULTIUSER MIMO RECEIVERS: FROM THE WORST-CASE TO PROBABILISTICALLY CONSTRAINED DESIGNS
}

\author{
Sergiy A. Vorobyov Yue Rong Alex B. Gershman \\ Communication Systems Group, Darmstadt University of Technology, Merckstr. 25, 64283 Darmstadt, Germany
}

\begin{abstract}
Two related problems of the design of robust adaptive beamformers and multiuser multiple-input multiple-output (MIMO) receivers are considered. A popular recent solution to these problems is based on the worst-case performance optimization. Unfortunately, in practical applications the actual worst case occurs with a very low probability and, as a result, the worst-case based designs may be overly conservative. As a less conservative alternative to the worst-case designs, the so-called probabilistically constrained designs are introduced. The latter approach guarantees that the distortionless response constraint is satisfied for a mismatched array response with a certain selected probability. Improved flexibility and performance of the robust probabilistically constrained designs with respect to the worst-case designs are illustrated via simulations.
\end{abstract}

\section{INTRODUCTION}

A recent popular approach to robust adaptive beamforming and robust multiuser MIMO receiver design is based on the worst-case performance optimization [1]-[4]. The techniques developed using this approach aim to optimize the performance assuming that the array operates under the worst conditions. However, the actual worst operational conditions may occur in practice with a very low probability. Therefore, the techniques based on the worst-case approach may be overly conservative and may lead to unnecessary performance losses. Hence, obtaining less conservative robust alternatives is of great interest. This paper introduces such an alternative probabilistically constrained approach.

Our paper is organized as follows. In the next section we provide an overview of robust adaptive beamforming and robust multiuser MIMO receiver design. Section 3 presents a short description of the worst-case approach in application to the considered problems. The probabilistically constrained approach is presented in Section 4. Simulation results are given in Section 5.

\section{BACKGROUND}

\subsection{Adaptive Beamforming}

The output of a narrowband beamformer is given by

$$
y(t)=\boldsymbol{w}^{H} \boldsymbol{x}(t)
$$

where $t$ is the sample index, $\boldsymbol{x}(t)$ is the $M \times 1$ complex vector of array observations, $\boldsymbol{w}$ is the $M \times 1$ complex vector of beamformer weights, $M$ is the number of array sensors, and $(\cdot)^{H}$ denotes the Hermitian transpose.
The optimal weight vector $\boldsymbol{w}$ in (1) can be obtained by maximizing the signal-to-interference-plus-noise ratio (SINR), or, in the finite sample case, by solving the following optimization problem

$$
\min _{\boldsymbol{w}} \boldsymbol{w}^{H} \hat{\boldsymbol{R}} \boldsymbol{w} \quad \text { subject to } \quad \boldsymbol{w}^{H} \boldsymbol{a}=1
$$

where $\boldsymbol{a}$ is the signal steering vector, $\hat{\boldsymbol{R}}=\frac{1}{J} \sum_{t=1}^{J} \boldsymbol{x}(t) \boldsymbol{x}(t)^{H}$ is the $M \times M$ sample covariance matrix, and $J$ is the number of snapshots available. The solution to (2) is given by the well known sample matrix inversion (SMI) based minimum variance distortionless response (MVDR) beamformer $\boldsymbol{w}=\left(\boldsymbol{a}^{H} \hat{\boldsymbol{R}}^{-1} \boldsymbol{a}\right)^{-1} \hat{\boldsymbol{R}}^{-1} \boldsymbol{a}$.

\subsection{Multiuser MIMO Receivers}

A problem related to adaptive beamforming is the multiuser MIMO receiver design problem. To establish a link between these two problems, let us consider an uplink multiuser MIMO communication system. Assuming that the transmitters have the same number of antennas and encode information-bearing symbols using the same orthogonal space-time block code (OSTBC) [4], the received signal can be written as

$$
\boldsymbol{Y}=\sum_{i=1}^{I} \boldsymbol{X}_{i} \boldsymbol{H}_{i}+\boldsymbol{V}
$$

where $\boldsymbol{Y} \triangleq\left[\boldsymbol{y}^{T}(1) \cdots \boldsymbol{y}^{T}(T)\right]^{T}, \boldsymbol{X}_{i} \triangleq\left[\boldsymbol{x}_{i}^{T}(1) \cdots \boldsymbol{x}_{i}^{T}(T)\right]^{T}$, and $\boldsymbol{V} \triangleq\left[\boldsymbol{v}^{T}(1) \cdots \boldsymbol{v}^{T}(T)\right]^{T}$ are the matrices of the received signals, transmitted signals of the $i$ th transmitter, and white Gaussian noise, respectively, $\boldsymbol{H}_{i}$ is the $N \times M$ complex channel matrix between the $i$ th transmitter and the receiver, $\boldsymbol{y}(t) \triangleq\left[y_{1}(t) \cdots y_{M}(t)\right], \boldsymbol{x}_{i}(t) \triangleq$ $\left[x_{i, 1}(t) \cdots x_{i, N}(t)\right]$, and $\boldsymbol{v}(t) \triangleq\left[v_{1}(t) \cdots v_{M}(t)\right]$ are the row vectors of the received signals, transmitted signals of the $i$ th transmitter, and noise, respectively, $I$ is the number of transmitters, $N$ is the number of transmit antennas, $T$ is the block length, and $(\cdot)^{T}$ denotes the transpose. The model (3) can be rewritten as [4]

$$
\underline{\boldsymbol{Y}}=\sum_{i=1}^{I} \boldsymbol{A}\left(\boldsymbol{H}_{i}\right) \underline{\boldsymbol{s}_{i}}+\underline{\boldsymbol{V}}
$$

where $\boldsymbol{s}_{i} \triangleq\left[s_{i, 1} \cdots s_{i, K}\right]^{T}$ is the complex vector of informationbearing symbols of the $i$ th transmitter prior to space-time encoding, $\underline{\boldsymbol{P}} \triangleq\left[\operatorname{vec}(\operatorname{Re}\{\boldsymbol{P}\})^{\mathrm{T}} \operatorname{vec}(\operatorname{Im}\{\boldsymbol{P}\})^{\mathrm{T}}\right]^{\mathrm{T}}$ for any matrix $\boldsymbol{P}, \operatorname{vec}(\cdot)$ is the vectorization operator stacking all columns of a matrix on top of each other, $\boldsymbol{A}\left(\boldsymbol{H}_{i}\right)$ is the $2 M T \times 2 K$ real matrix defined as

$$
\begin{aligned}
\boldsymbol{A}\left(\boldsymbol{H}_{i}\right) & \triangleq\left[\underline{\boldsymbol{C}_{1} \boldsymbol{H}_{i}} \cdots \underline{\boldsymbol{C}_{K} \boldsymbol{H}_{i}} \underline{\boldsymbol{D}_{1} \boldsymbol{H}_{i}} \cdots \underline{\boldsymbol{D}_{K} \boldsymbol{H}_{i}}\right] \\
& \triangleq\left[\boldsymbol{a}_{1}\left(\boldsymbol{H}_{i}\right) \cdots \boldsymbol{a}_{2 K}\left(\boldsymbol{H}_{i}\right)\right]
\end{aligned}
$$


and $\boldsymbol{C}_{1}, \ldots, \boldsymbol{C}_{K}, \boldsymbol{D}_{1}, \ldots, \boldsymbol{D}_{K}$ are the OSTBC matrices [4].

Assuming that the first user is the user of interest, the estimate of the data vector $\underline{\hat{\boldsymbol{s}}_{1}}$ at the output of a linear receiver can be expressed as [4]

$$
\underline{\hat{\boldsymbol{s}}_{1}}=\boldsymbol{W}^{T} \underline{\boldsymbol{Y}}
$$

where $\boldsymbol{W}=\left[\boldsymbol{w}_{1} \cdots \boldsymbol{w}_{2 K}\right]$ is the $2 M T \times 2 K$ real matrix of the receiver weight coefficients, and $\boldsymbol{w}_{k}$ is the $2 M T \times 1$ real weight vector that corresponds to decoding the $k$ th entry of $\boldsymbol{s}_{1}$. The problem of finding the matrix $\boldsymbol{W}$ that separates the signals from different users is conceptually similar to the problem of finding a weight vector $\boldsymbol{w}$ in (1). This similarity provides an opportunity to design the matrix $\boldsymbol{W}$ using the MV principle. Specifically, each entry of $\underline{s}_{1}$ can be estimated by minimizing the receiver output power while preserving a unity gain for this entry of $\underline{s}_{1}$. It is also important to incorporate self-interference cancellation using additional zero-forcing constraints $\boldsymbol{w}_{k}^{T} \boldsymbol{a}_{l}\left(\boldsymbol{H}_{1}\right)=0$ for all $l \neq k$ [4]. Then, the problem of linear receiver design can be written as [4]

$$
\min _{\boldsymbol{W}} \operatorname{tr}\left\{\boldsymbol{W}^{T} \hat{\boldsymbol{R}} \boldsymbol{W}\right\} \quad \text { subject to } \quad \boldsymbol{A}^{T}\left(\boldsymbol{H}_{1}\right) \boldsymbol{W}=\boldsymbol{I}_{2 K}
$$

where $\boldsymbol{I}_{m}$ and $\operatorname{tr}\{\cdot\}$ denote the $m \times m$ identity matrix and the trace of a matrix, respectively, $\hat{\boldsymbol{R}}=\frac{1}{J} \sum_{j=1}^{J} \underline{\boldsymbol{Y}_{j}}{\underline{\boldsymbol{Y}_{j}}}^{T}$ is the sample estimate of the $2 M T \times 2 M T$ full rank covariance matrix $\boldsymbol{R} \triangleq$ $\mathrm{E}\left\{\underline{\boldsymbol{Y}} \underline{\boldsymbol{Y}}^{T}\right\}$ of the vectorized data, $\boldsymbol{Y}_{j}$ is the $j$ th received data block, and $\mathrm{E}\{\cdot\}$ denotes the statistical expectation. The solution to the MV problem (7) can be obtained in a similar way as the solution to (2), and is given by $\boldsymbol{W}=\hat{\boldsymbol{R}}^{-1} \boldsymbol{A}\left(\boldsymbol{H}_{1}\right)\left(\boldsymbol{A}^{T}\left(\boldsymbol{H}_{1}\right) \hat{\boldsymbol{R}}^{-1} \boldsymbol{A}\left(\boldsymbol{H}_{1}\right)\right)^{-1}$.

\section{WORST-CASE BASED DESIGNS}

\subsection{Robust Adaptive Beamformer Design}

An essential shortcoming of the MVDR beamformer is that it is not robust against a mismatch between the presumed and actual signal steering vectors $\boldsymbol{a}$ and $\tilde{\boldsymbol{a}}$, respectively. In [1] and [2], the actual (mismatched) steering vector $\tilde{\boldsymbol{a}}$ has been explicitly modelled as

$$
\tilde{a}=\boldsymbol{a}+\boldsymbol{\delta} \neq \boldsymbol{a}
$$

where $\delta$ denotes an unknown complex vector which describes the effect of steering vector errors (the mismatch vector). It has been assumed that $\delta$ is an unknown deterministic vector that is bounded in its norm by some known positive constant $\|\boldsymbol{\delta}\| \leq \varepsilon$, where $\|\cdot\|$ denotes the Euclidian (Frobenius) norm of a vector (matrix). The design of robust adaptive beamformer based on the worst-case approach boils down to solving the MVDR problem for the worst-case steering vector [1]

$$
\min _{\boldsymbol{w}} \boldsymbol{w}^{H} \hat{\boldsymbol{R}} \boldsymbol{w} \quad \text { subject to } \min _{\|\boldsymbol{\delta}\| \leq \varepsilon}\left|\boldsymbol{w}^{H} \tilde{\boldsymbol{a}}\right| \geq 1 .
$$

The problem (9) can be equivalently rewritten as a so-called secondorder cone programming (SOCP) problem and efficiently solved using interior-point optimization techniques [1], or the Newton-type numerical procedure [2]. The complexity of solving such problem is comparable to that of the SMI beamformer.

\subsection{Robust Multiuser MIMO Receiver Design}

It can be seen from (7) that the MV receiver requires the CSI of the user of interest. However, in practice, it is unrealistic to obtain the exact CSI at the receiver. The only quantity available at the receiver is the estimate $\hat{\boldsymbol{H}}_{1}$, which represents a mismatched copy of $\boldsymbol{H}_{1}$. Thus, similarly to (8) the actual channel can be modelled as

$$
\boldsymbol{H}_{1}=\hat{\boldsymbol{H}}_{1}+\boldsymbol{\Delta}_{1} \neq \hat{\boldsymbol{H}}_{1}
$$

where $\boldsymbol{\Delta}_{1}$ is the matrix of CSI errors.

Similarly to the SMI beamformer, an essential shortcoming of the MV receiver in (7) is that it is not robust against CSI errors. To add robustness to this MV receiver, it has been assumed in [4] that $\boldsymbol{\Delta}_{1}$ is an unknown deterministic matrix whose norm is bounded by some known positive constant, that is, $\left\|\boldsymbol{\Delta}_{1}\right\| \leq \varepsilon$. Using the notations of (5), it can be shown that for any OSTBC $\left\|\Delta_{1}\right\|=$ $\left\|\boldsymbol{e}_{k}\right\|$ for all $k=1, \ldots, 2 K$, where $\boldsymbol{e}_{k} \triangleq \boldsymbol{a}_{k}\left(\boldsymbol{H}_{1}\right)-\boldsymbol{a}_{k}\left(\hat{\boldsymbol{H}}_{1}\right)$ [4]. Then, using the worst-case approach, the robust modification of (7) should minimize the output power subject to the constraint that the distortionless response is maintained for the worst-case $\boldsymbol{a}_{k}(\hat{\boldsymbol{H}}+$ $\boldsymbol{\Delta})$, and subject to the worst-case zero-forcing constraints for selfinterference. Then, the corresponding optimization problem can be written as [4]

$$
\begin{aligned}
& \min _{\boldsymbol{w}_{k}} \boldsymbol{w}_{k}^{T} \hat{\boldsymbol{R}} \boldsymbol{w}_{k} \text { subject to } \min _{\left\|\boldsymbol{e}_{k}\right\| \leq \varepsilon} \boldsymbol{w}_{k}^{T}\left(\boldsymbol{a}_{k}\left(\hat{\boldsymbol{H}}_{1}\right)+\boldsymbol{e}_{k}\right) \geq 1 \\
& \max _{\left\|\boldsymbol{E}_{k}\right\| \leq \eta} \|\left(\boldsymbol{B}_{k}^{T}+\boldsymbol{E}_{k}^{T}\right) \boldsymbol{w}_{k} \leq \xi_{k}
\end{aligned}
$$

where the $2 M T \times(2 K-1)$ matrices $\boldsymbol{B}_{k}$ and $\boldsymbol{E}_{k}$ are defined as $\boldsymbol{B}_{k} \triangleq\left[\boldsymbol{a}_{1}\left(\hat{\boldsymbol{H}}_{1}\right) \ldots \boldsymbol{a}_{k-1}\left(\hat{\boldsymbol{H}}_{1}\right) \boldsymbol{a}_{k+1}\left(\hat{\boldsymbol{H}}_{1}\right) \ldots \boldsymbol{a}_{2 K}\left(\hat{\boldsymbol{H}}_{1}\right)\right]$ and $\boldsymbol{E}_{k} \triangleq\left[\boldsymbol{e}_{1} \ldots \boldsymbol{e}_{k-1} \boldsymbol{e}_{k+1} \ldots \boldsymbol{e}_{2 K}\right]$, respectively, $\xi_{k}$ is the value that limits the contribution of self-interference in the uncertainty region $\left\|\boldsymbol{E}_{k}\right\| \leq \eta$, and $\eta=\varepsilon \sqrt{2 K-1}$ [4]. The problem (11) can be equivalently written as a SOCP problem [4], and solved with the complexity comparable to the complexity of the MV receiver (7).

\subsection{Probability of the Worst Case: Numerical Example}

To characterize the probability of the worst case, let us consider a numerical example for the beamformer (9). In this case, the worst case mismatch vector can be written as: $\boldsymbol{\delta}_{\mathrm{W}}=-\boldsymbol{w} \varepsilon e^{j \phi} /\|\boldsymbol{w}\|$, where $\phi=\operatorname{angle}\left\{\boldsymbol{w}^{H} \boldsymbol{a}\right\}$ [1]. Considering a scenario with Rician propagation medium, where the presumed signal steering vector is the plane wave with the nominal direction-of-arrival (DOA) $\theta_{0}$ while the actual steering vector corresponds to a spread source around $\theta_{0}$, the actual mismatch vector $\boldsymbol{\delta}$ can be modelled as $\boldsymbol{\delta}=$ $\frac{\sigma_{\delta}}{\sqrt{L}} \sum_{l=1}^{L} e^{j \psi_{l}} \boldsymbol{a}\left(\theta_{0}+\theta_{l}\right)$, where $\sigma_{\delta}^{2}$ characterizes the mismatch (scattering) power, $L$ is the number of nonline-of-sight (NLOS) components due to scattering, $\psi_{l}$ is the phase shift parameter of the $l$ th NLOS component, and $\theta_{l}$ is the angle shift of $l$ th NLOS component with respect to the nominal DOA. We denote the norm of deviation of the actual mismatch vector from the worst case mismatch vector as $z=\left\|\boldsymbol{\delta}_{\mathrm{W}}-\boldsymbol{\delta}\right\|$.

For calculating $\delta_{\mathrm{W}}$, we choose the signal-to-noise ratio (SNR) and interference-to-noise ratio (INR) to be equal to $15 \mathrm{~dB}$ and $40 \mathrm{~dB}$, respectively, $M=10, \varepsilon=3$, and $J=100$. For calculating $\delta$, the parameters $\theta_{l}$ are independently drawn in each simulation run from a uniform random generator with zero mean and unit variance, and the parameters $\psi_{l}$ are independently and uniformly drawn from the half-interval $[0,2 \pi)$ in each run.

Fig. 1 shows the histogram $p(z)$ computed from 1000 trials for $\delta$. This figure confirms our conjecture that the worst-case designs may be overly conservative: note that the probability of occurrence of the worst-case mismatch is nearly zero, and most of mismatch vectors are far away from the worst-case mismatch vector. 


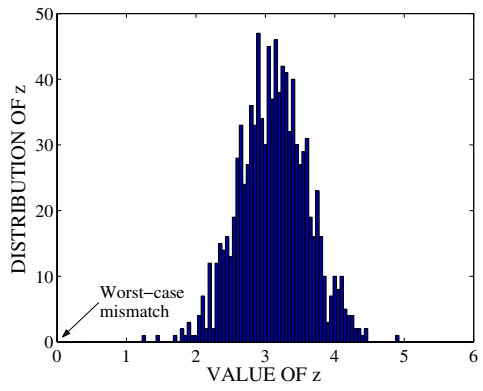

Fig. 1. The histogram of $p(z)$.

\section{PROBABILISTICALLY CONSTRAINED DESIGNS}

\subsection{Robust Adaptive Beamformer Design}

Assuming that the mismatch $\delta$ is an unknown random vector with known probability density function, the robust formulation of the adaptive beamformer based on the probabilistically constrained approach can be written as

$$
\min _{\boldsymbol{w}} \boldsymbol{w}^{H} \hat{\boldsymbol{R}} \boldsymbol{w} \quad \text { subject to } \operatorname{Pr}\left\{\left|\boldsymbol{w}^{H} \tilde{\boldsymbol{a}}\right| \geq 1\right\} \geq p
$$

where $p$ is a certain probability value, and $\operatorname{Pr}\{\cdot\}$ stands for the probability operator. The problem (12) belongs to a class of chanceor probability-constrained stochastic programming problems [5]. It aims to minimize the beamformer output power subject to the constraint that the probability of the distortionless response to the signal with mismatched steering vector is lower-bounded by a certain selected value of $p$.

In general, the problem (12) is mathematically intractable. However, it can be approximately solved if we assume a specific analytic form for the probability operator $\operatorname{Pr}\{\cdot\}$ [6]. Assuming that $\delta$ is drawn from a complex circularly symmetric Gaussian distribution with zero mean and covariance matrix $C_{\delta}$, i.e., $\delta \sim \mathcal{C N}\left(\mathbf{0}_{M}, C_{\delta}\right)$, it is easy to verify that $\boldsymbol{w}^{H}(\boldsymbol{a}+\boldsymbol{\delta}) \sim \mathcal{C} \mathcal{N}\left(\boldsymbol{w}^{H} \boldsymbol{a},\left\|\boldsymbol{C}_{\boldsymbol{\delta}}^{1 / 2} \boldsymbol{w}\right\|^{2}\right)$. Hence, the random variable $\left|\boldsymbol{w}^{H}(\boldsymbol{a}+\boldsymbol{\delta})\right|$ has Rician distribution. To avoid complications related to this distribution, we approximate the constraint in (12) by the following constraints: $\operatorname{Pr}\left\{\left|\operatorname{Re}\left\{\boldsymbol{w}^{H} \tilde{\boldsymbol{a}}\right\}\right| \geq\right.$ $\beta\} \geq p$ and $\operatorname{Pr}\left\{\left|\operatorname{Im}\left\{\boldsymbol{w}^{H} \tilde{\boldsymbol{a}}\right\}\right| \geq \beta\right\} \geq p$, where $\beta=1 / \sqrt{2}$ is the solution to the equation $1=\left|\boldsymbol{w}^{H} \tilde{\boldsymbol{a}}\right|^{2}=\operatorname{Re}\left\{\boldsymbol{w}^{H} \tilde{\boldsymbol{a}}\right\}^{2}+\operatorname{Im}\left\{\boldsymbol{w}^{H} \tilde{\boldsymbol{a}}\right\}^{2}=$ $2 \beta^{2}$. Then, the optimization problem (12) can be approximated by

$$
\begin{aligned}
& \min _{\boldsymbol{w}} \boldsymbol{w}^{H} \hat{\boldsymbol{R}} \boldsymbol{w} \\
& \text { subject to } \operatorname{Pr}\left\{\left|\operatorname{Re}\left\{\boldsymbol{w}^{H} \tilde{\boldsymbol{a}}\right\}\right| \geq 1 / \sqrt{2}\right\} \geq p \\
& \operatorname{Pr}\left\{\left|\operatorname{Im}\left\{\boldsymbol{w}^{H} \tilde{\boldsymbol{a}}\right\}\right| \geq 1 / \sqrt{2}\right\} \geq p
\end{aligned}
$$

where $\operatorname{Re}\left\{\boldsymbol{w}^{H} \tilde{\boldsymbol{a}}\right\}$ and $\operatorname{Im}\left\{\boldsymbol{w}^{H} \tilde{\boldsymbol{a}}\right\}$ are real Gaussian random variables with the means $\operatorname{Re}\left\{\boldsymbol{w}^{H} \boldsymbol{a}\right\}$ and $\operatorname{Im}\left\{\boldsymbol{w}^{H} \boldsymbol{a}\right\}$, respectively, and the variance $\left\|C_{\delta}^{1 / 2} \boldsymbol{w}\right\|^{2} / 2$. The first constraint in (13) can be equivalently rewritten as

$$
\begin{gathered}
\operatorname{Pr}\left\{\left|\operatorname{Re}\left\{\boldsymbol{w}^{H} \tilde{\boldsymbol{a}}\right\}\right| \geq 1 / \sqrt{2}\right\}=1-\operatorname{Pr}\left\{\left|\operatorname{Re}\left\{\boldsymbol{w}^{H} \tilde{\boldsymbol{a}}\right\}\right| \leq 1 / \sqrt{2}\right\} \\
=1-\left[\operatorname{Pr}\left\{\operatorname{Re}\left\{\boldsymbol{w}^{H} \tilde{\boldsymbol{a}}\right\} \leq 1 / \sqrt{2}\right\}-\operatorname{Pr}\left\{\operatorname{Re}\left\{\boldsymbol{w}^{H} \tilde{\boldsymbol{a}}\right\} \leq-1 / \sqrt{2}\right\}\right] \\
=1-\frac{1}{2}\left[\operatorname{erf}\left(\frac{\sqrt{1 / 2}-\operatorname{Re}\left\{\boldsymbol{w}^{H} \boldsymbol{a}\right\}}{\left\|\boldsymbol{C}_{\boldsymbol{\delta}}^{1 / 2} \boldsymbol{w}\right\|}\right)\right. \\
\left.-\operatorname{erf}\left(\frac{-\sqrt{1 / 2}-\operatorname{Re}\left\{\boldsymbol{w}^{H} \boldsymbol{a}\right\}}{\left\|\boldsymbol{C}_{\boldsymbol{\delta}}^{1 / 2} \boldsymbol{w}\right\|}\right)\right]
\end{gathered}
$$

where $\operatorname{erf}(\cdot)$ denotes the standard error function for Gaussian distribution. The same steps are also applicable to the second constraint Then we can write a deterministic equivalent to the problem (13) as

$$
\begin{aligned}
\min _{\boldsymbol{w}} \boldsymbol{w}^{H}\left(\hat{\boldsymbol{R}}+\gamma \boldsymbol{I}_{M}\right) \boldsymbol{w} \\
\text { subject to } \quad \operatorname{erf}\left(\frac{-\sqrt{1 / 2}-\operatorname{Re}\left\{\boldsymbol{w}^{H} \boldsymbol{a}\right\}}{\left\|\boldsymbol{C}_{\boldsymbol{\delta}}^{1 / 2} \boldsymbol{w}\right\|}\right) \\
-\operatorname{erf}\left(\frac{\sqrt{1 / 2}-\operatorname{Re}\left\{\boldsymbol{w}^{H} \boldsymbol{a}\right\}}{\left\|\boldsymbol{C}_{\boldsymbol{\delta}}^{1 / 2} \boldsymbol{w}\right\|}\right) \geq 2(p-1) \\
\quad \operatorname{erf}\left(\frac{-\sqrt{1 / 2}-\operatorname{Im}\left\{\boldsymbol{w}^{H} \boldsymbol{a}\right\}}{\left\|\boldsymbol{C}_{\boldsymbol{\delta}}^{1 / 2} \boldsymbol{w}\right\|}\right) \\
-\operatorname{erf}\left(\frac{\sqrt{1 / 2}-\operatorname{Im}\left\{\boldsymbol{w}^{H} \boldsymbol{a}\right\}}{\left\|\boldsymbol{C}_{\boldsymbol{\delta}}^{1 / 2} \boldsymbol{w}\right\|}\right) \geq 2(p-1)
\end{aligned}
$$

where an additional fixed diagonal loading (DL) (with the DL factor $\gamma$ ) is used to add the robustness against small sample size, and $p \in(0.5,1)$. The problem (15) is convex and belongs to a class of nonlinear programming (NLP) problems [7]. It can be efficiently solved using sequential quadratic programming (SQP) technique. The latter technique is an iterative approach in which each search direction is the solution of a particular quadratic programming (QP) subproblem [7]. The computational complexity of solving QP subproblems is higher than the complexity of solving SOCP problems.

\subsection{Robust Multiuser MIMO Receiver Design}

The probabilistically constrained design for the robust multiuser receiver corresponds to obtaining the receiver coefficient vector $\boldsymbol{w}_{k}$ for the $k$ th entry of $\underline{s}_{1}$ as the solution to the following probabilistically constrained optimization problem

$$
\begin{array}{cl}
\min _{\boldsymbol{w}_{k}, \boldsymbol{\xi}} & \boldsymbol{w}_{k}^{T} \hat{\boldsymbol{R}} \boldsymbol{w}_{k}+\|\boldsymbol{\xi}\|^{2} \\
\text { s.t. } & \operatorname{Pr}\left\{\boldsymbol{w}_{k}^{T}\left(\boldsymbol{a}_{k}\left(\hat{\boldsymbol{H}}_{1}\right)+\boldsymbol{e}_{k}\right) \geq 1\right\} \geq p, \\
& \operatorname{Pr}\left\{\sigma_{1}\left|\boldsymbol{w}_{k}^{T}\left(\boldsymbol{a}_{l}\left(\hat{\boldsymbol{H}}_{1}\right)+\boldsymbol{e}_{l}\right)\right| \leq \xi_{l}\right\} \geq p, \\
& l=1, \ldots, 2 K, \quad l \neq k
\end{array}
$$

where $\boldsymbol{\xi}=\left[\xi_{1}, \ldots, \xi_{k-1}, \xi_{k+1}, \cdots, \xi_{2 K}\right]^{T}$ is the $(2 K-1) \times 1$ vector whose values limit the contribution of self-interference, and $\sigma_{1}$ is the standard deviation of the waveform of the desired user.

It is important to note that the probability bound $p$ in the problems (16) determines the amount of channel mismatch that is allowed at the receiver and should be selected according to the quality of service (QoS) requirements. In contrast to the worst-case based formulation (11), the formulation (16) maintains the distortionless response for $k$ th entry of $\underline{s}_{1}$ and suppresses self-interference probabilistically rather than deterministically. Note that the probabilistic distortionless response constraint in (16) can be also referred to as a non-outage probability constraint.

Similarly to (13), the problem (16) can be converted into its convex deterministic equivalent. Specifically, assuming that $\left[\boldsymbol{\Delta}_{i}\right]_{n, m} \sim$ $\mathcal{C N}\left(0, \sigma_{h}^{2}\right)$ and $p \in(0.5,1)$, the first constraint in (16) can be easily converted into a second-order cone (SOC) constraint [8]

$$
\sigma_{h}\left\|\left(\boldsymbol{I}_{2 M} \otimes \boldsymbol{G}_{k}^{T}\right) \boldsymbol{w}_{k}\right\| \leq \frac{\boldsymbol{w}_{k}^{T} \boldsymbol{a}_{k}\left(\hat{\boldsymbol{H}}_{1}\right)-1}{\operatorname{erf}^{-1}(2 p-1)}
$$


where $\boldsymbol{G}_{k}=\left\{\begin{array}{cl}\boldsymbol{C}_{k}, & k=1, \ldots, K \\ \operatorname{Im}\left\{\boldsymbol{D}_{k-K}\right\}, & k=K+1, \ldots, 2 K\end{array} \quad\right.$ and $\otimes$ stands for the Kronecker product. The last $2 K-1$ constraints in (16) can be rewritten as the following deterministic convex nonlinear constraints

$$
\begin{aligned}
& \operatorname{erf}\left(\frac{\xi_{l}-\sigma_{1} \boldsymbol{w}_{k}^{T} \boldsymbol{a}_{l}\left(\hat{\boldsymbol{H}}_{1}\right)}{\sigma_{h} \sigma_{1}\left\|\left(\boldsymbol{I}_{2 M} \otimes \boldsymbol{G}_{l}^{T}\right) \boldsymbol{w}_{k}\right\|}\right) \\
& -\operatorname{erf}\left(\frac{-\xi_{l}-\sigma_{1} \boldsymbol{w}_{k}^{T} \boldsymbol{a}_{l}\left(\hat{\boldsymbol{H}}_{1}\right)}{\sigma_{h} \sigma_{1}\left\|\left(\boldsymbol{I}_{2 M} \otimes \boldsymbol{G}_{l}^{T}\right) \boldsymbol{w}_{k}\right\|}\right) \geq 2 p \\
& l=1, \ldots, 2 K, \quad l \neq k
\end{aligned}
$$

or can be approximated by the following SOC constraints [8]

$$
\begin{aligned}
& \frac{\sigma_{1}^{2}}{1-p} \boldsymbol{w}_{k}^{T}\left(\boldsymbol{a}_{l}\left(\hat{\boldsymbol{H}}_{1}\right) \boldsymbol{a}_{l}^{T}\left(\hat{\boldsymbol{H}}_{1}\right)+\frac{\sigma_{h}^{2}}{2}\left(\boldsymbol{I}_{2 M} \otimes \boldsymbol{G}_{l} \boldsymbol{G}_{l}^{T}\right)\right) \boldsymbol{w}_{k} \leq \xi_{l}^{2} \\
& l=1, \ldots, 2 K, \quad l \neq k .
\end{aligned}
$$

Using (18), the problem (16) can be equivalently written as NLP problem, and using (19) it can be approximated by the following SOCP problem

$$
\begin{aligned}
\min _{\boldsymbol{w}_{k}, \tau} & \tau \\
\text { subject to } & \left\|\boldsymbol{Z}_{k} \boldsymbol{w}_{k}\right\| \leq \tau \\
& \sigma_{h}\left\|\left(\boldsymbol{I}_{2 M} \otimes \boldsymbol{G}_{k}^{T}\right) \boldsymbol{w}_{k}\right\| \leq \frac{\boldsymbol{w}_{k}^{T} \boldsymbol{a}_{k}\left(\hat{\boldsymbol{H}}_{1}\right)-1}{\operatorname{erf}^{-1}(2 p-1)}
\end{aligned}
$$

where $\hat{\boldsymbol{R}}+\boldsymbol{Q}_{k}=\boldsymbol{Z}_{k}^{T} \boldsymbol{Z}_{k}$ is the Cholesky factorization of $\hat{\boldsymbol{R}}+\boldsymbol{Q}_{k}$, $\tau$ is a new variable such that $\left\|\boldsymbol{Z}_{k} \boldsymbol{w}_{k}\right\| \leq \tau$, and

$$
\boldsymbol{Q}_{k} \triangleq \frac{\sigma_{1}^{2}}{1-p} \sum_{l=1, l \neq k}^{2 K}\left[\boldsymbol{a}_{l}\left(\hat{\boldsymbol{H}}_{1}\right) \boldsymbol{a}_{l}^{T}\left(\hat{\boldsymbol{H}}_{1}\right)+\frac{\sigma_{h}^{2}}{2}\left(\boldsymbol{I}_{2 M} \otimes \boldsymbol{G}_{l} \boldsymbol{G}_{l}^{T}\right)\right]
$$

The approximate SOCP problem (20) is computationally more attractive than the NLP problem.

\section{SIMULATIONS}

In this section we consider a simulation example in application to the robust multiuser MIMO receiver design. An uplink cellular communication system with multiple transmitters is simulated. Throughout the simulations, we assume a single receiver equipped with $M=$ 8 antennas. The MIMO channel between the $i$ th transmitter and the receiver is assumed to be quasi-static Rayleigh flat fading with $\left[\boldsymbol{H}_{i}\right]_{n, m} \sim \mathcal{C N}(0,1)$. The channel mismatch $\boldsymbol{\Delta}_{i}$ is assumed to be independent on $\boldsymbol{H}_{i}$ with $\left[\boldsymbol{\Delta}_{i}\right]_{n, m} \sim \mathcal{C N}\left(0, \sigma_{h}^{2}\right)$. We set $\sigma_{h}^{2}=0.1$.

The following receivers are compared in terms of symbol error rates (SERs): the SOCP-based approximation of the probabilistically-constrained receiver (20) denoted as PC-SOCP, the worstcase based SOCP receiver (WC-SOCP) (11), and the DL version of the MV receiver (7) (DLMV), where the DL factor $10 \sigma_{v}^{2}$ is chosen, and $\sigma_{v}^{2}$ is the noise variance. The probability $p$ in the proposed robust receivers is set to 0.99 . For the worst-case based receiver, $\varepsilon=7 \sigma_{h}$, as suggested in [4]. 300 Monte Carlo runs are used to obtain each curve.

A scenario with $I=4$ transmitters, where each transmitter is equipped with $N=3$ antennas, is simulated. The half rate ( $K=4, T=8$ ) OSTBC from [9] is employed. The INR is equal

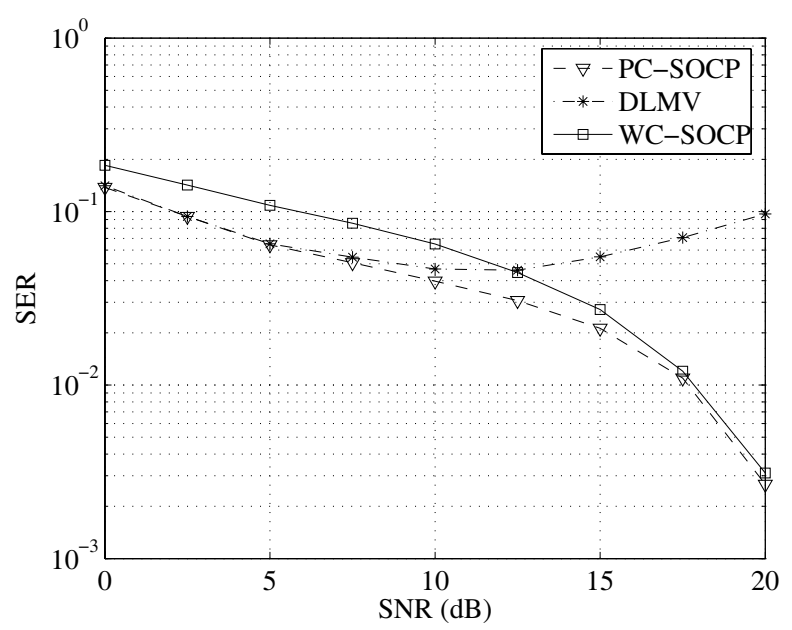

Fig. 2. SER versus SNR.

to $20 \mathrm{~dB}$ and the QPSK modulation scheme is used. Fig. 2 shows the SER performance of the aforementioned methods versus SNR when $J$ is equal to 130 . It clearly demonstrates that the proposed probabilistically-constrained robust receiver consistently enjoys the best performance as compared with the other methods tested. Moreover, the proposed receiver outperforms the worst-case based receiver, especially in low and medium SNR regions.

\section{REFERENCES}

[1] S. A. Vorobyov, A. B. Gershman, and Z.-Q. Luo, "Robust adaptive beamforming using worst-case performance optimization: A solution to the signal mismatch problem," IEEE Trans. Signal Processing, vol. 51, pp. 313-324, Feb. 2003.

[2] R. G. Lorenz and S. P. Boyd, "Robust minimum variance beamforming," IEEE Trans. Signal Processing, vol. 53, pp. 16841696, May 2005.

[3] J. Li, P. Stoica, and Z. Wang, "On robust Capon beamforming and diagonal loading," IEEE Trans. Signal Processing, vol. 51, pp. 1702-1715, July 2003.

[4] Y. Rong, S. Shahbazpanahi, and A. B. Gershman, "Robust linear receivers for space-timeblock coded multiaccess MIMO systems with imperfect channel state information," IEEE Trans. Signal Processing, vol. 53, pp. 3081-3090, Aug. 2005.

[5] A. Prékopa, Stochastic Programming. Dordrecht, Netherlands: Kluwer Academic Publishers, 1995.

[6] S. A. Vorobyov, Y. Rong, and A. B. Gershman, "Robust adaptive beamforming using probability-constrained optimization," Proc. IEEE SSP Workshop, Bordeaux, France, July 2005.

[7] D. P. Bertsekas, Nonlinear Programming. Belmont, Athena Scientific, 1995.

[8] Y. Rong, S. A. Vorobyov, and A. B. Gershman, "Robust linear receiver design for multi-access space-time block coded MIMO systems using stochastic optimization," Proc. IEEE SSP Workshop, Bordeaux, France, July 2005.

[9] V. Tarokh, H. Jafarkhani, and A. R. Calderbank, "Space-time block codes from orthogonal designs," IEEE Trans. Information Theory, vol. 45, pp. 1456-1467, July 1999. 